\title{
The high-temperature behavior of defect hydrogen species in quartz: Implications for hydrogen isotope studies
}

\author{
Kevin Grant, ${ }^{1, *}$ Sarah A. GleEson,${ }^{2, \uparrow} \uparrow$ AND STEVe RobertS ${ }^{1}$ \\ ${ }^{1}$ School of Ocean and Earth Science, Southampton Oceanography Centre, University of Southampton, Empress Dock, Southampton SO14 3ZH, U.K. \\ ${ }^{2}$ School of Earth Sciences, University of Leeds, Leeds LS2 9JT, U.K.
}

\begin{abstract}
A micro-infrared spectroscopic study of hydrothermal vein quartz known to have anomalous $\delta \mathrm{D}$ signatures has identified two hydrogen reservoirs. In samples that generate an isotopic signature in accordance with that anticipated under the accepted model of quartz crystallization, submicroscopic aggregates of liquid water are the dominant hydrous species. Samples which generate an anomalous $\delta \mathrm{D}$ signature contain, in addition to liquid water, structurally incorporated hydrous species associated with impurity cations.

Infrared spectra obtained during in situ stepped heating experiments, coupled with infrared analysis at $25{ }^{\circ} \mathrm{C}$, demonstrate that hydrogen liberated between 300 and $500{ }^{\circ} \mathrm{C}$ is chiefly molecular, liquid water. Hydrogen liberated at temperatures greater than $500{ }^{\circ} \mathrm{C}$ is dominantly that associated with specific structurally incorporated cation defects. Since both defect hydrogen and molecular water are contemporaneously incorporated from the precipitating medium during crystallization, we propose that irregular $\delta \mathrm{D}$ signatures, released following decrepitation at temperatures greater than 500 ${ }^{\circ} \mathrm{C}$, are due to isotopically fractionated hydrogen released from interstitial $\mathrm{OH}$ defect sites in the quartz structure.

$\delta \mathrm{D}$ signatures obtained from stoichiometrically anhydrous minerals are generally interpreted under the assumption that the hydrogen measured comes uniquely from decrepitated fluid inclusions. Instead, we suggest that $\delta \mathrm{D}$ ratios obtained from hydrothermal quartz reflect a sum of the contributions made by individual hydrogen reservoirs, each with a potentially distinctive $\delta \mathrm{D}$ signature. Thus, if the overall $\delta \mathrm{D}$ signature is attributed entirely to fluid inclusion phases, the nature of the precipitating fluid may be misinterpreted. Hydrogen extracted as molecular water at between 300 and $500{ }^{\circ} \mathrm{C}$ provides a true reflection of the hydrothermal solution associated with crystal precipitation.
\end{abstract}

